\title{
Response to bevacizumab combination chemotherapy of malignant pleural effusions associated with non-squamous non-small-cell lung cancer
}

\author{
KATSUHIRO MASAGO ${ }^{1,2}$, DAICHI FUJIMOTO ${ }^{3}$, SHIRO FUJTA $^{1}$, AKITO HATA ${ }^{1}$, REIKO KAJI ${ }^{1}$, \\ KYOKO OHTSUKA $^{1,4}$, CHIYUKI OKUDA ${ }^{1}$, JUMPEI TAKESHITA ${ }^{1}$ and NOBUYUKI KATAKAMI ${ }^{1}$ \\ ${ }^{1}$ Division of Integrated Oncology, Institute of Biomedical Research and Innovation Hospital, Kobe, Hyogo 650-0047; \\ ${ }^{2}$ Department of Pathology and Molecular Diagnostics, Aichi Cancer Center, Nagoya, Aichi 464-8681; \\ ${ }^{3}$ Department of Respiratory Medicine, Kobe City Medical Center General Hospital, Kobe, Hyogo 650-0047; \\ ${ }^{4}$ Department of Respiratory Medicine, Osaka City University, Osaka, Osaka 558-8585, Japan
}

Received September 18, 2014; Accepted October 29, 2014

DOI: $10.3892 / \mathrm{mco} .2014 .457$

\begin{abstract}
Malignant pleural effusion (MPE) is a common complication of lung cancer with devastating consequences. Since vascular endothelial growth factor (VEGF) has been implicated in MPE, we hypothesized that bevacizumab, an anti-VEGF antibody, may be effective against MPE in patients with non-small-cell lung cancer (NSCLC). We analysed the records of 21 patients treated for NSCLC-associated MPE between February, 2010 and August, 2013 who consequently underwent bevacizumab combination chemotherapy at the Institute of Biomedical Research and Innovation Hospital. The results were retrospectively analysed using case records and radiographic imaging records. Three patients exhibited complete response of the pleural effusion to bevacizumab treatment, 8 patients achieved a partial response (PR) and 6 patients showed no response. When efficacy was assessed by the response of the measurable primary or metastatic lesions to the treatment, 5 patients achieved a PR, 13 patients had stable disease and 3 patients exhibited progressive disease. The response rate (RR) of the pleural effusion to the antibody treatment was $71.4 \%$ and the overall RR of measurable lesions was $23.8 \%$. The median time-to-response for pleural effusion was 132 days. In conclusion, this study demonstrated a high RR to bevacizumab combination therapy for the MPE associated with non-squamous NSCLC. Therefore, bevacizumab therapy may be considered a therapeutic option for patients with non-squamous NSCLC who develop MPE.
\end{abstract}

Correspondence to: Dr Katsuhiro Masago, Division of Integrated Oncology, Institute of Biomedical Research and Innovation Hospital, 2-2 Minatojima-minamimachi, Chuo-ku, Kobe, Hyogo 650-0047, Japan

E-mail: masago@fbri.org

Key words: bevacizumab, malignant pleural effusion, non-small-cell lung cancer, vascular endothelial growth factor

\section{Introduction}

Malignant pleural effusion (MPE) is a common and devastating complication of lung cancer, with $15 \%$ of lung cancer patients presenting with pleural effusion at the time of initial diagnosis, whereas half of the patients develop pleural effusion at a later stage of the disease $(1,2)$. MPE may cause significant dyspnea, cough and chest pain. There are currently several management options for MPE, including chemical pleurodesis with chest tubes or medical thoracoscopy, video-assisted thoracic surgery, pleuroperitoneal shunts and chronic indwelling pleural catheter. However, all these management options have certain disadvantages (3).

Vascular endothelial growth factor (VEGF) is the founding member of an expanding family of endothelial cell growth factors. VEGF, also known as vascular permeability factor, has been implicated in MPE (4). VEGF is a powerful inducer of vascular permeability; it is 50,000 times more potent than histamine (5). In addition, VEGF expression may be induced by nearly all cell types and is often overexpressed in lung cancer cells $(6,7)$.

Bevacizumab is a humanized monoclonal antibody against VEGF with demonstrated antitumour effects in lung cancer cell lines and animal models (8). Results from in vitro studies have demonstrated that this monoclonal antibody is able to effectively neutralize almost all VEGF-mediated activities (9). It was previously shown that the administration of an anti-VEGF antibody lead to a significant reduction in the amount of pleural fluid within the first week following intrapleural injection of talc or nitrate (10). This antibody was also successfully used for the treatment of recurrent pleural effusions in a patient with amyloidosis (11). Bevacizumab in combination with carboplatin/paclitaxel improved overall survival (OS) and is currently approved in the United States and Japan for use in patients with recurrent or metastatic non-squamous, non-small cell lung cancer (NSCLC) chemotherapy (12).

Therefore, we hypothesized that the administration of the anti-VEGF antibody bevacizumab may be beneficial as a treatment option for MPE in NSCLC patients. In this 
study, we retrospectively analysed the efficacy of combination chemotherapies that included bevacizumab against NSCLC-associated MPE.

\section{Materials and methods}

Patient selection. We analysed records from 21 patients with advanced NSCLC and MPE who consequently underwent bevacizumab combination chemotherapy between February, 2010 and August, 2013 at the Institute of Biomedical Research and Innovation Hospital, Kobe, Hyogo, Japan. Data were retrospectively collected from case records and radiographic imaging records. Written informed consent regarding bevacizumab therapy was acquired from all patients. This study was approved by the Institutional Review Board of our institute.

Evaluation of efficacy. Measurable lesions and the size of the MPE were determined by computed tomography (CT) scan prior to bevacizumab combination chemotherapy. Tumour response was evaluated by CT every 4-8 weeks according to the Response Evaluation Criteria in Solid Tumours Committee (13). If a patient was documented to exhibit a complete response (CR) or a partial response (PR), a confirmation with a second scan was required after an additional 4 weeks. The response of each tumour was recorded as the best tumour response observed over the entire course of treatment. Response rate (RR) was defined as $\mathrm{CR}+\mathrm{PR}$.

The size of the pleural effusion was defined as follows: Massive, effusion volume $>75 \%$ of the hemithorax; large, effusion volume $50-75 \%$ of the hemithorax; moderate, effusion volume $25-50 \%$ of the hemithorax; and small, effusion volume $<25 \%$ of the hemithorax. The objective response of the MPE was evaluated using chest X-rays and CT scans and a method similar to a previous report (14). CR was defined as the complete disappearance of pleural fluid for 4 weeks. PR was defined as a distinguishable decrease for 4 weeks. No response was defined as failure to meet the abovementioned criteria. CR was evaluated only by CT scans. The time-to-response was defined as the period between the initiation of bevacizumab therapy and the first detectable reduction of the pleural effusion volume by $\mathrm{CT}$ or chest $\mathrm{X}$-ray. Time-to-response was calculated using only patients with either a CR or a PR; patients that showed no response were not included in this calculation.

\section{Results}

Patient characteristics. First, we reviewed the demographics of the patients included in the study. The patient characteristics are summarized in Table I. All the patients were Japanese and included 11 men (52\%) and 10 women (48\%), with a median age of 46 years (range, 30-86 years). Eleven patients (52\%) were never-smokers and 10 patients $(48 \%)$ were current or former smokers. All the patients had stage IV adenocarcinoma according to the 7th edition of the TNM classification (15). The majority of the patients $(12 / 21,57.1 \%)$ had an Eastern Cooperative Oncology Group performance status (ECOG PS) of 2. EGFR mutations were detected in 13 of the 21 patients (61.9\%) and anaplastic lymphoma kinase (ALK) rearrangement was detected in 3 cases (14.7\%).
The patients were grouped based on the size of the pleural effusion; 7 patients (33.3\%) had a moderate effusion size, 6 patients $(28.5 \%)$ had large effusions, whereas 4 patients (19.1\%) each had massive and small effusions. A total of 15 patients (71.4\%) had received prior chemotherapy. The standard dose of bevacizumab $(15 \mathrm{mg} / \mathrm{kg})$ was administered to all the patients. In combination with bevacizumab, the patients received one of the following regimens: carboplatin plus paclitaxel $(n=6)$, erlotinib $(n=5)$, vinorelbine $(n=4)$, carboplatin plus pemetrexed $(n=2)$, docetaxel $(n=2)$, or paclitaxel $(n=2)$.

Response to treatment. We assessed the response of the patients to the combination therapy including bevacizumab by reviewing the change in the effusion volume over the course of the treatment. Of the 21 patients, 7 achieved a CR, 8 had a PR and 6 patients did not show a response. We next investigated the patient assessments of the primary or metastatic lesion response to the combination therapy. A total of 5 patients exhibited a PR, 13 patients had stable disease and 3 patients showed progressive disease (Table II). The RR of the pleural effusion to therapy was $71.4 \%$ and the overall RR of measurable lesions to therapy was $23.8 \%$. Of the 6 patients who exhibited no response, 5 had no increase in the effusion volume compared to the original measurement. Of the 15 patients who achieved a CR or PR regarding the pleural effusion, 3 patients (25\%) did not exhibit a re-accumulation of pleural effusion following completion of the treatment.

\section{Discussion}

The goal of our study was to review the RR of MPE to a combination therapy that included bevacizumab. Overall, we observed that $23.8 \%$ of measurable lesions showed a response. This tumour RR is similar to those of previous reports examining a high dose bevacizumab combination therapy, which reported RRs of $\sim 30 \%(12,16)$. However, this study also demonstrated a high RR of NSCLC-associated MPE to the high-dose bevacizumab combination therapy; $71.4 \%$ of MPE has some measurable decrease in volume.

In a number of patients with NSCLC-associated MPE, standard systemic chemotherapy was proven to be ineffective $(3,18)$. Kitamura et al reported that bevacizumab in combination with chemotherapy was highly effective for the management of MPE in patients with non-squamous NSCLC (18). Combined intrapleural therapy with bevacizumab and cisplatin was found to be effective and safe in managing NSCLC-associated MPE, with a curative efficacy of $83.33 \%$ (19). According to another study, intense combination chemotherapy including cisplatin, ifosfamide, irinotecan and recombinant human granulocyte colony-stimulating factor support achieved high RRs of the pleural effusions and measurable lesions (58.8 and 73.5\%, respectively) (14). Notably, our study demonstrated a higher RR of pleural effusion to a combinatorial therapy that included a high dose of bevacizumab.

Several studies demonstrated that VEGF is associated with the formation of pleural effusion, the effusion size and poor patient survival (20-24). It was also reported that VEGF receptor phosphorylation inhibited the formation of malignant effusion in mice with lung adenocarcinomas. This result was attributed to reduced vascular permeability (25). 
Table I. Patient characteristics $(n=21)$.

Characteristics

Age (years)

Range

Median

Gender

Male

Female

Smoking status

Never-smoker

Current or former-smoker

ECOG PS ${ }^{\mathrm{a}}$

1

2

Histology

Adenocarcinoma

Large-cell neuroendocrine cell carcinoma

Non-small-cell lung cancer NOS

Stage

IV

EGFR status

Mutation $^{\mathrm{b}}$

Wild-type

Unknown

ALK rearrangement

Positive

Negative

Unknown (number of EGFR mutants)

Size of pleural effusion

Small

Moderate

Large

Massive

Prior chemotherapy

Yes

No

Chemotherapy schema

Carboplatin + paclitaxel + bevacizumab

Erlotinib + bevacizumab

Vinorelbine + bevacizumab

Carboplatin + pemetrexed + bevacizumab

Docetaxel + bevacizumab

Paclitaxel + bevacizumab

Patient no.

30-86

46

11

10

11

10

9

12

19

1

1

21

100.0

13

7

1

61.9

33.3

4.8

3

14.7

7

$11(5)$

33.3

52.0

19.1

33.3

28.5

19.1

4

15

71.4

6

28.6

28.5

23.8

18.9

9.6

9.6

9.6

aPerformance status evaluated prior to the administration of bevacizumab. ${ }^{\mathrm{b}} \mathrm{EGFR}$ mutation-positive; exon 19 del, exon 21 L858R, L861Q. SD, standard deviation; ECOG PS, Eastern Cooperative Oncology Group performance status; NOS, not otherwise specified; EGFR, epidermal growth factor receptor gene; ALK, anaplastic lymphoma kinase gene.

Mesiano et al reported that the production of ascitic fluid induced by intraperitoneal inoculation of ovarian cancer cells was almost completely inhibited by neutralizing antibodies that block the action of VEGF (26). Considering the results from those in vitro studies, anti-VEGF therapy may be more effective for malignant effusion rather than for primary tumours. Recombinant human endostatin (Endostar) reduced the expression of VEGF-A and MPE in mice with Lewis lung 
Table II. Response to bevacizumab-containing treatment.

Tumour response (no.) $)^{\mathrm{a}}$

Response of pleural effusion (no.) ${ }^{\mathrm{b}}$

\section{Partial \\ response}

Stable

disease
Progressive

disease

3
0
2
5

$\begin{array}{rr}3 & 1 \\ 7 & 1 \\ 3 & 1 \\ 13 & 3\end{array}$

${ }^{\mathrm{a}}$ Tumour response rate, $23.8 \%$. ${ }^{\mathrm{b}}$ Response rate of pleural effusion, $71.4 \%$.

carcinoma (27). This result may explain the differences we observed between the response of pleural effusions and that of measurable lesions to bevacizumab.

In this study, all the patients received the standard dose of bevacizumab $(15 \mathrm{mg} / \mathrm{kg})$. Pichelmayer et al reported data on 4 patients with malignant effusions who received bevacizumab therapy (11). In that study, 2 patients who received low-dose bevacizumab (5 or $10 \mathrm{mg} / \mathrm{kg}$ ) achieved no significant reduction of the malignant effusions. By contrast, 2 patients who received the standard dose $(15 \mathrm{mg} / \mathrm{kg})$ achieved a reduction of the malignant effusion. The results of those studies suggest that treatment of malignant effusion with bevacizumab may require administration of the standard dose.

There are currently several management options for MPE, such as chemical pleurodesis with chest tubes, medical thoracoscopy, video-assisted thoracic surgery, pleuroperitoneal shunts and chronic indwelling pleural catheter $(3,17)$. Chemical pleurodesis is the most commonly used modality for managing MPE. However, patients with a multi-loculated effusion, trapped lung, or bronchial obstruction are unlikely to benefit from intrapleural therapy. Typically, such patients may be treated with systemic chemotherapy. Therefore, intrapleural therapy is not ideal and should be reserved for patients who are refractory to or meet the exclusion criteria for systemic chemotherapy. Based upon our results, bevacizumab therapy alone may be a treatment option for non-squamous NSCLC patients with MPE and poor performance status.

This study had certain limitations. First, there are no standard criteria to evaluate response in patients with MPE. Therefore, we used the response criteria reported by a previous study (14). Second, we were unable to confirm negative cytological findings in the pleural effusions following bevacizumab therapy, as a thoracentesis was difficult in cases where a CR or PR was observed. However, a confirmation of the response, which requires over 4 weeks and a RR of $67.0 \%$ were considered satisfactory. Finally, this study was conducted entirely by retrospectively reviewing electronic medical charts. A prospective study may improve our understanding of the potential and efficacy of anti-VEFG therapy.

In conclusion, this study demonstrated a high RR to bevacizumab combination therapy of the MPE associated with non-squamous NSCLC. Therefore, bevacizumab therapy may be a management option for patients with MPE associated with non-squamous NSCLC.

\section{References}

1. Anderson CB, Philpott GW and Ferguson TB: The treatment of malignant pleural effusions. Cancer 33: 916-922, 1974.

2. Memon A and Zawadzki ZA: Malignant effusions: diagnostic evaluation and therapeutic strategy. Curr Probl Cancer 5: 1-30, 1981.

3. Thomas JM and Musani AI: Malignant pleural effusions: a review. Clin Chest Med 34: 459-471, 2013.

4. Zebrowski BK, Yano S, Liu W, et al: Vascular endothelial growth factor levels and induction of permeability in malignant pleural effusions. Clin Cancer Res 5: 3364-3368, 1999.

5. Brown LF, Detmar M, Claffey K, et al: Vascular permeability factor/vascular endothelial growth factor: a multifunctional angiogenic cytokine. EXS 79: 233-269, 1997.

6. Takahama M, Tsutsumi M, Tsujiuchi T, et al: Enhanced expression of Tie2, its ligand angiopoietin-1, vascular endothelial growth factor, and CD31 in human non-small cell lung carcinomas. Clin Cancer Res 5: 2506-2510, 1999.

7. Ferrara N: Vascular endothelial growth factor: molecular and biological aspects. Curr Top Microbiol Immunol 237: 1-30, 1999.

8. Bertino EM and Otterson GA: Benefits and limitations of antiangiogenic agents in patients with non-small cell lung cancer. Lung Cancer 70: 233-246, 2010.

9. Wang Y, Fei D, Vanderlaan M and Song A: Biological activity of bevacizumab, a humanized anti-VEGF antibody in vitro. Angiogenesis 7: 335-345, 2004.

10. Ribeiro SC, Vargas FS, Antonangelo L, et al: Monoclonal anti-vascular endothelial growth factor antibody reduces fluid volume in an experimental model of inflammatory pleural effusion. Respirology 14: 1188-1193, 2009.

11. Pichelmayer O, Zielinski $\mathrm{C}$ and Raderer M: Response of a nonmalignant pleural effusion to bevacizumab. N Engl J Med 353: 740-741, 2005.

12. Sandler A, Gray R, Perry MC, et al: Paclitaxel-carboplatin alone or with bevacizumab for non-small-cell lung cancer. N Engl J Med 355: 2542-2550, 2006.

13. Therasse P, Arbuck SG, Eisenhauer EA, et al: New guidelines to evaluate the response to treatment in solid tumors. European Organization for Research and Treatment of Cancer, National Cancer Institute of the United States, National Cancer Institute of Canada. J Natl Cancer Inst 92: 205-216, 2000.

14. Fujita A, Takabatake H, Tagaki S and Sekine K: Combination chemotherapy in patients with malignant pleural effusions from non-small cell lung cancer : cisplatin, ifosfamide, and irinotecan with recombinant human granulocyte colony-stimulating factor support. Chest 119: 340-343, 2001.

15. Goldstraw P, Crowley J, Chansky K, et al; International Association for the Study of Lung Cancer International Staging Committee; Participating Institutions: The IASLC Lung Cancer Staging Project: proposals for the revision of the TNM stage groupings in the forthcoming (seventh) edition of the TNM classification of malignant tumours. J Thorac Oncol 2: 706-714, 2007.

16. Johnson DH, Fehrenbacher L, Novotny WF, et al: Randomized phase II trial comparing bevacizumab plus carboplatin and paclitaxel with carboplatin and paclitaxel alone in previously untreated locally advanced or metastatic non-small-cell lung cancer. J Clin Oncol 22: 2184-2191, 2004. 
17. Musani AI: Treatment options for malignant pleural effusion Curr Opin Pulm Med 15: 380-387, 2009.

18. Kitamura K, Kubota K, Ando M, et al: Bevacizumab plus chemotherapy for advanced non-squamous non-small-cell lung cancer with malignant pleural effusion. Cancer Chemother Pharmacol 71: 457-461, 2012.

19. Du N,Li X,Li F, et al: Intrapleural combination therapy with bevacizumab and cisplatin for non-small cell lung cancer-mediated malignant pleural effusion. Oncol Rep 29: 2332-2340, 2013.

20. Verheul HM, Hoekman K, Jorna AS, Smit EF and Pinedo HM: Targeting vascular endothelial growth factor blockade: ascites and pleural effusion formation. Oncologist 1: 45-50, 2000.

21. Yanagawa H, Takeuchi E, Suzuki Y, Ohmoto Y, Bando H and Sone S: Vascular endothelial growth factor in malignant pleural effusion associated with lung cancer. Cancer Immunol Immunother 48: 396-400, 1999.

22. Yano S, Shinohara H, Herbst RS, et al: Production of experimental malignant pleural effusions is dependent on invasion of the pleura and expression of vascular endothelial growth factor/vascular permeability factor by human lung cancer cells. Am J Pathol 157: 1893-1903, 2000.
23. Cheng D, Rodriguez RM, Perkett EA, et al: Vascular endothelial growth factor in pleural fluid. Chest 116: 760-765, 1999.

24. Hsu IL, Su WC, Yan JJ, Chang JM and Lai WW: Angiogenetic biomarkers in non-small cell lung cancer with malignant pleural effusion: correlations with patient survival and pleural effusion control. Lung Cancer 65: 371-376, 2009.

25. Yano S, Herbst RS, Shinohara H, et al: Treatment for malignant pleural effusion of human lung adenocarcinoma by inhibition of vascular endothelial growth factor receptor tyrosine kinase phosphorylation. Clin Cancer Res 6: 957-965, 2000.

26. Mesiano S, Ferrara N and Jaffe RB: Role of vascular endothelial growth factor in ovarian cancer: inhibition of ascites formation by immunoneutralization. Am J Pathol 153: 1249-1256, 1998.

27. Ma X, Yao Y, Yuan D, et al: Recombinant human endostatin endostar suppresses angiogenesis and lymphangiogenesis of malignant pleural effusion in mice. PLoS One 7: e53449, 2012. 